\title{
Blood dosimetry from a single measurement of the whole body radioiodine retention in patients with differentiated thyroid carcinoma
}

\author{
Heribert Hänscheid, Michael Lassmann, Markus Luster, Richard T Kloos ${ }^{1}$ \\ and Christoph Reiners
}

Department of Nuclear Medicine, University of Würzburg, Oberdürrbacher Straße 6, D-97080 Würzburg, Germany

${ }^{1}$ Divisions of Endocrinology and Nuclear Medicine, Departments of Internal Medicine and Radiology, The Ohio State University, 446 McCampbell Hall, 1581 Dodd Drive Columbus, OH 43210-1296, USA

(Correspondence should be addressed to H Hänscheid; Email: haenscheid @ nuklearmedizin.uni-wuerzburg.de)

\begin{abstract}
A simple method is presented to estimate the radiation-absorbed dose to the blood after radioiodine administration from a single external measurement of the whole-body retention in patients suffering from differentiated thyroid cancer. The blood dose is calculated applying the formalism of the Medical International Radiation Dose Committee under the assumptions that whole-body activity decays exponentially and that $14 \%$ of the whole-body residence time can be attributed to the blood. Accuracy and applicability of the method were tested based on data from 29 assessments, 18 pre-therapeutic tracer studies, and 11 ablation therapies, with whole-body and blood-retention measurements over at least 4 days. The mean of the absolute deviations between estimates and actual blood doses was found to be $14 \%$, if external whole-body counting was performed on day 1 or 2 after radioiodine administration. This simple formalism is: 1) applicable to pre-therapeutic dosimetry for remnant ablation or treatment of metastases in a blood dose-based treatment concept and 2) applicable to blood-dose estimates after radioiodine therapy to determine radiation exposure. When combined with a measurement of the whole body retention 1 or 2 days after radioiodine administration this single time-point method closely approximates the classic, yet much more labor intensive multi-day dosimetry that measures both blood and whole-body activities.
\end{abstract}

Endocrine-Related Cancer (2009) 16 1283-1289

\section{Introduction}

Radiation absorbed dose to the red bone marrow, a critical organ in the therapy of differentiated thyroid carcinoma with ${ }^{131} \mathrm{I}$ (radioiodine), cannot be measured directly. As radioiodine concentration is comparable in blood and most organs (Kolbert et al. 2007), and is believed to be similar in red marrow (Sgouros 2005), the absorbed dose to the blood seems to be a good firstorder approximation of the radiation absorbed dose to the hematopoietic system and a better means to quantify exposure from therapy than the total amount of activity administered.

Blood dosimetry was introduced by Benua et al. (1962) in a study published in 1962. They found that radioiodine therapy is safe if the blood dose is restricted to $<2 \mathrm{~Gy}$ (200 $\mathrm{rad}$ ), while keeping the whole-body retention $<4.4 \mathrm{GBq}(120 \mathrm{mCi})$ at $48 \mathrm{~h}$, and the pulmonary uptake at $24 \mathrm{~h}<3 \mathrm{GBq}(80 \mathrm{mCi})$ (Benua et al. 1962, Benua \& Leeper 1986).

Most commonly, differentiated thyroid carcinoma is treated with empiric standard activities, a concept with obvious disadvantages. Radiation exposure from fixed activities is very heterogeneous. Depending principally on the patient's size and renal clearance, the calculated blood absorbed dose per unit of activity administered can differ by more than a factor of 5 (Hänscheid et al. 2006). Especially in older patients treated during hypothyroidism, the blood dose frequently exceeds 
the 2 Gy (200 rad) limit even after administration of activities of $7.4 \mathrm{GBq}(200 \mathrm{mCi})$ or less (Tuttle et al. 2006). On the other hand, a low absorbed dose to the blood might predict reduced radioiodine availability for target tissue uptake and, therefore, a low absorbed dose to the target tissue. Compartment model calculations indicate an almost linear relation between the blood residence time, which is the major determinant of the blood dose, and the radiation dose to the target tissue (Hänscheid et al. 2006). A high renal clearance resulting in a low blood dose must be expected to provide unfavorable conditions for radioiodine accumulation in the target tissue. This can be compensated for by increasing the treatment activity.

Individualized patient-specific therapy is ideally based on a pre-therapeutic dosimetry of both red marrow and target dose per activity administered and provides information on the activity necessary to eliminate all lesions or whether an effective tumor dose can be reached without exceeding the tolerable dose to the bone marrow. ${ }^{123} \mathrm{I}$ and ${ }^{131} \mathrm{I}$ are not ideal for such a dosimetry. ${ }^{131}$ I potentially induces stunning (Lassmann et al. 2004), the half-life of ${ }^{123} \mathrm{I}$ is too short and both nuclides require information on the target mass, the S-factor of self-irradiation, and the homogeneity of the activity concentration within the target tissue. Until supposedly superior procedures like ${ }^{124}$ I PET-based 3D dosimetry (Sgouros et al. 2004) become generally available blood-based dosimetry seems to be the best means to individualize therapy and to increase the safety of the treatment.

At present, blood dosimetry prior to radioiodine therapy is not recommended in guidelines to be part of standard operational procedures except in high-dose therapies targeting the blood dose at $2 \mathrm{~Gy}$ (200 rad) (Lassmann et al. 2008), and it is not practiced at most medical centers. Even in specialized centers it is often not routinely performed, but rather reserved for the minority of patients requiring high dose therapies or for other unusual circumstances such as renal failure or pediatric patients.

Two factors impede dosimetry based therapy:

1) studies to investigate whether the administration of individually determined activities is more efficient than therapy with standard activities are difficult to perform and, since the original work published by Benua et al. (1962), no study has demonstrated the clinical benefits of a dosimetry-based treatment;

2) complete blood dosimetry is laborious and resource consuming, and requires whole-body and blood measurements over 4 days or longer. Efforts to avoid (Thomas et al. 1993), reduce blood sampling (Traino et al. 2004) or to adapt the therapeutic activity in case of markedly low or high $48 \mathrm{~h}$ whole-body retention (Sisson et al. 2003) have failed to promote routine performance of dosimetry.

As an advancement of the method presented in Thomas et al. (1993), the present approach provides blood dose estimates from a single external measurement of the whole body retention 1 or 2 days after radioiodine administration and enables blood dosebased therapy for routine remnant ablation, as well as treatment of metastatic disease, all with minimal effort.

\section{Materials and methods}

\section{Absorbed dose to the blood}

An equation to determine the mean blood absorbed dose $\bar{D}_{\text {blood }}$ per unit of administered activity $\left(A_{0}\right)$ based on the generally accepted formalism of the Medical International Radiation Dose Committee was recently published (Lassmann et al. 2008):

$$
\begin{aligned}
& \frac{\overline{\mathrm{D}}_{\text {blood }}}{\mathrm{A}_{0}}\left(\frac{\mathrm{Gy}}{\mathrm{GBq}}\right) \\
& =108 \times \tau_{\text {ml of blood }}(\mathrm{h})+\frac{0.0188}{(\mathrm{wt}(\mathrm{kg}))^{2 / 3}} \\
& \quad \times \tau_{\text {total body }}(\mathrm{h})
\end{aligned}
$$

where $\tau_{\text {total body }}$, total body residence time; $\tau_{\mathrm{ml} \text { of blood, }}$ residence time in a $\mathrm{ml}$ of whole blood; and wt, patient's weight in $\mathrm{kg}$.

The first addend in Equation 1 accounts for the contribution from $\beta$-radiation assuming energy absorption of $187 \mathrm{keV}$ per decay in the blood. The second addend accounts for decays outside the blood contributing to the blood dose by penetrating radiation with an $S$-value depending on the patient's weight: $\mathrm{S}_{\text {blood } \leftarrow \gamma \text { total body }}=0.0188 / \mathrm{wt}^{2 / 3} \mathrm{~Gy} /(\mathrm{GBq} \times \mathrm{h})$. Results deduced with Equation 1 are in good agreement with the calculations based on the formalism used in Benua et al. (1962).

A method to estimate blood dose from external whole-body counting without blood sampling was proposed by Thomas et al. (1993). The authors found in a study with 49 dosimetric assessments that $14-17 \%$ (range 3-25\%) of the whole-body residence time can be attributed to the blood. The percentage deduced in the present investigation is $13 \pm 3 \%$ (range $7-21 \%$ ). 
The corresponding figure as deduced from a recently published study (Hänscheid et al. 2006) is 14士3\% (range 8-24\%).

The relation can be expressed as:

$\tau_{\text {ml of blood }}(\mathrm{h})=\frac{0.14}{\mathrm{BLV}(\mathrm{ml})} \times \tau_{\text {total body }}(\mathrm{h})$

The individual blood volume BLV can be estimated from the patient's weight wt $(\mathrm{kg})$ and height $\mathrm{ht}(\mathrm{cm})$ (Retzlaff et al. 1969) to be $\mathrm{BLV}=31.9 \times \mathrm{ht}+26.3$ $\times w t-2402$ for males and $\mathrm{BLV}=56.9 \times \mathrm{ht}+14.1$ $\times w t-6460$ for females.

Equations 1 and 2 can be combined to Equation 3 being applicable to estimate the blood dose from the whole-body residence time:

$$
\begin{aligned}
& \frac{\mathrm{D}_{\text {blood }}}{\mathrm{A}_{0}}(\mathrm{mGy} / \mathrm{MBq}) \\
& \quad=\left(\frac{15.12}{\mathrm{BLV}(\mathrm{ml})}+\frac{0.0188}{(\mathrm{wt}(\mathrm{kg}))^{2 / 3}}\right) \times \tau_{\text {total body }}(\mathrm{h})
\end{aligned}
$$

Sisson et al. (2003) proposed to use the $48 \mathrm{~h}$ wholebody retention measured in a diagnostic assessment to adapt the activity in the subsequent radioiodine therapy in case of markedly low or high $48 \mathrm{~h}$ whole-body uptake. As a step further, a blood dose estimate from a single measurement of the whole-body retention can be deduced if the retention $R(t)$ at $t$ hours after the radioiodine administration is taken to be representative for the total-body residence time according to:

$\tau_{\text {total body }}=-\frac{\mathrm{t}}{\ln (\mathrm{R}(\mathrm{t}))}$

The blood absorbed dose becomes

$$
\begin{aligned}
& \frac{\mathrm{D}_{\text {blood }}}{\mathrm{A}_{0}}(\mathrm{mGy} / \mathrm{MBq}) \\
& \quad=-\left(\frac{15.12}{\mathrm{BLV}(\mathrm{ml})}+\frac{0.0188}{(\mathrm{wt}(\mathrm{kg}))^{2 / 3}}\right) \times \frac{\mathrm{t}(\mathrm{h})}{\ln (\mathrm{R}(\mathrm{t}))}
\end{aligned}
$$

or in traditional units

$$
\begin{aligned}
& \frac{D_{\text {blood }}}{A_{0}}(\mathrm{rad} / \mathrm{mCi}) \\
& \quad=-\left(\frac{56}{\mathrm{BLV}(\mathrm{ml})}+\frac{0.7}{(\mathrm{wt}(\mathrm{kg}))^{2 / 3}}\right) \times \frac{\mathrm{t}(\mathrm{h})}{\ln (\mathrm{R}(\mathrm{t}))}
\end{aligned}
$$

\section{Patients}

In order to test the accuracy of the predictions calculated with Equation 5, an intentionally heterogeneous collection of three subgroups of patients suffering from differentiated thyroid cancer (age, 14-72 years; weight, 43-190 kg; blood volume BLV, 2837-9103 ml) were analyzed:

1) six patients participated in controlled studies including blood and remnant dosimetry totaling 12 courses of diagnostic application of $74 \mathrm{MBq}$ $(2 \mathrm{mCi})$ radioiodine prior to remnant ablation (Lassmann et al. 2004). Each patient was investigated twice, in hypothyroidism after thyroid hormone withdrawal and when euthyroid after stimulation with recombinant human TSH (rhTSH). The highest observed residence time in the target tissue was $23 \mathrm{~h}$ in a euthyroid female with maximum remnant uptake of $9 \%$ after $24 \mathrm{~h}$ (Lassmann et al. 2004);

2) six patients (three euthyroid, three hypothyroid) received diagnostic activities of $10 \mathrm{MBq}(0.3 \mathrm{mCi})$ in preparation for high-dose therapies. All of them showed only minor uptake in target tissue;

3) eleven patients participated in a study applying blood dosimetry after administration of nominal 3.7 GBq (100 mCi) for thyroid remnant ablation. All patients in this subgroup were hypothyroid.

Informed consent was given by every patient. All studies contributing data to the present investigation were approved by the local ethics committee and by German radiation protection authorities if legally required.

\section{Data evaluation}

All patients underwent whole-body retention measurements and blood sampling at nominal $2 \mathrm{~h}, 6 \mathrm{~h}, 24 \mathrm{~h}$, $48 \mathrm{~h}, 72 \mathrm{~h}$ (optional), and after 4-6 days. Whole-body measurements were performed as conjugate view (anterior and posterior) counts by scintillation camera imaging after administration of diagnostic activities for subgroups 1 and 2, or by use of a survey meter at a fixed distance of $2 \mathrm{~m}$ during therapy for subgroup 3 . Planar scintillation camera images were analyzed by extraction of count rates registered in regions of interest including the entire body.

Retention was calculated by normalization of the geometric mean of background corrected anterior and posterior counts to the initial measurement which was made $2 \mathrm{~h}$ after administration without interim micturition or defecation. The patients were asked to empty their urinary bladder prior to subsequent whole-body counts. Blood activity concentrations were measured in a calibrated well counter. Data integrity was assured 
by adequate quality control procedures as recommended in Lassmann et al. (2008).

The residence times in whole body $\tau_{\text {total body }}$ and blood $\tau_{\mathrm{ml} \text { of blood }}$ were determined by integrating biphasic decay curves fitted to the whole-body retention and blood activity concentration data, respectively, and actual blood dose values $a D$ were calculated with Equation 1 .

Blood dose estimates $e D$ according to Equation 5 from measured whole body retentions at nominal $6 \mathrm{~h}$, $24 \mathrm{~h}, 48 \mathrm{~h}$, and on day 3-6 after administration are compared with the $a D$ values in Fig. 1 and Table 1.

As an independent check, data from a multinational controlled study published in the supplemental data (available online) of Hänscheid et al. (2006) were analyzed. For 59 out of 63 patients listed in Hänscheid et al. (2006), information is sufficient to compare blood dose prediction $e D$ from the $48 \mathrm{~h}$ whole-body retention with the blood dose $a D$ as calculated with Equation 1 . Results are shown in Table 1 and Fig. 1C.

\section{Results and discussion}

The accuracy of estimates was found to be highest when whole body retention is measured on day 1 or day 2 after administration as illustrated in Fig. 1 and Table 1.
Assessments based on data gathered between 4.3 and $7.1 \mathrm{~h}$ after administration yielded ratios of estimate to actual dose $e D / a D$ ranging from 0.50 to 1.89 (Table 1). In one patient the retention at $4.0 \mathrm{~h}$ was measured to be $98 \%$ yielding a ratio of 16.7 .

The ratios $e D / a D$ as determined from the first wholebody retention measurement after at least 3 days ranged from 0.92 to 2.40 , with the median at 1.33 (Table 1). Late measurements tend to overestimate the dose (Fig. 1D), especially in patients with a high uptake and long effective half-life in target tissues.

The mean of the absolute deviations between estimate $e D$ and actual blood dose $a D$ is $13-14 \%$ if external whole-body counting is performed on day 1 or 2 after radioiodine administration. The median of the absolute deviations is even less with $11 \%$ on day 2 . $90 \%$ of the ratios $e D / a D$ of all data shown in Fig. 1B and $\mathrm{C}$ are within a range of unity $\pm 25 \%$ (min: 0.67 ; max: 1.51). A single outlier with $e D / a D=1.84$ was due to an obviously erroneous $48 \mathrm{~h}$ retention value which was measured to be higher than that after $24 \mathrm{~h}$ thus explaining the deviation of more than four S.D.'s.

Underestimation of the actual dose of $>25 \%$ occurred for two of the 29 assessments included in the present investigation when the estimate was
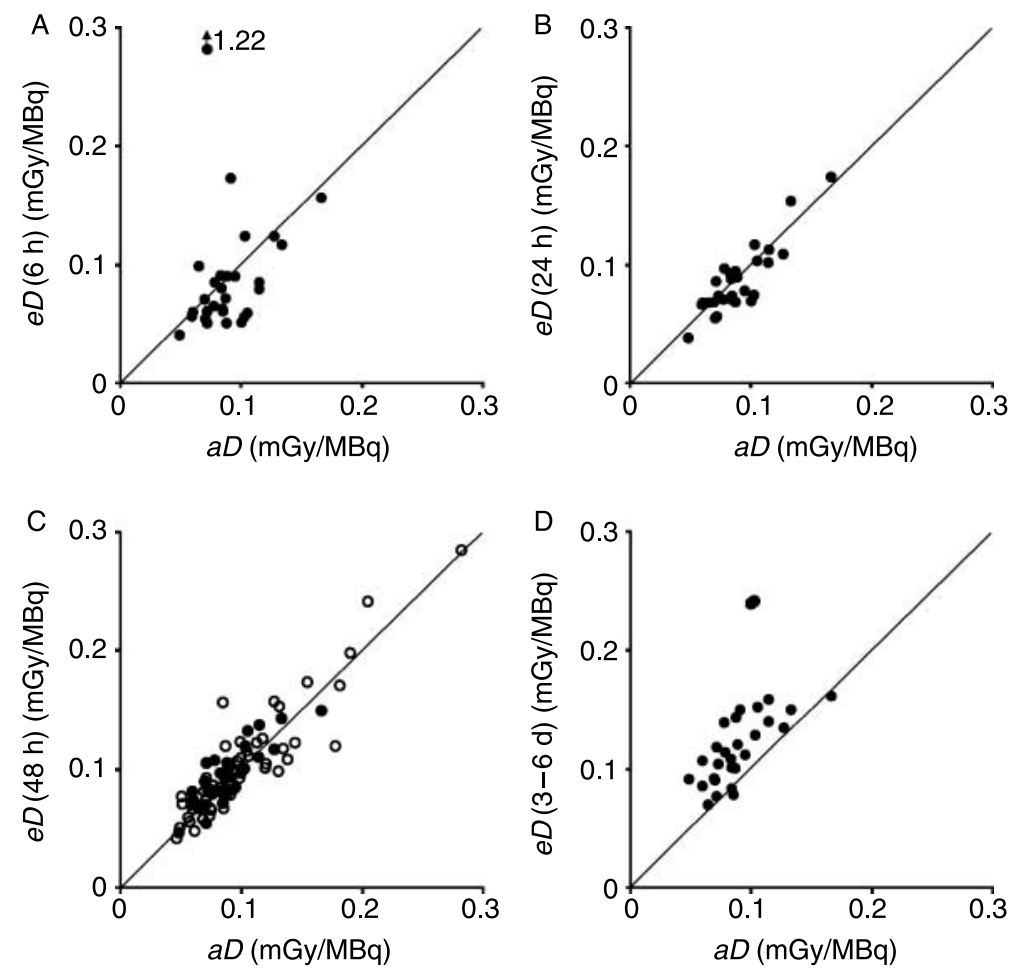

Figure 1 Blood dose estimates $e D(t)$ from single whole body retention measurements after $\mathrm{A}, t=6 \mathrm{~h} ; \mathrm{B}, t=24 \mathrm{~h} ; \mathrm{C}, t=48 \mathrm{~h} ;$ and $\mathrm{D}$, from the first measurement after at least 3 days versus actual blood dose $(a D)$. Filled dots represent measurements of patients included in the present investigation $(n=29)$, open circles in C are data from Hänscheid et al. (2006), $n=59$. 
Table 1 Statistics of deviations between actual blood doses $a D$ and estimates $e D$ based on a single whole body retention measurement. External data were taken from Hänscheid et al. (2006). The first measurement after at least 3 days for each patient was taken to deduce the $3-6$ days values

\begin{tabular}{llllll}
\hline & & Present investigation & & External data \\
\hline Time of measurement & $6 \mathrm{~h}^{\mathrm{a}}$ & $24 \mathrm{~h}^{\mathrm{c}}$ & $48 \mathrm{~h}$ & $3-6$ days & $48 \mathrm{~h}^{\mathrm{b}}$ \\
Number of patients included & 28 & 28 & 29 & 29 & 58 \\
Median of ratio $e D / a D$ & 0.85 & 0.99 & 1.04 & 1.33 & 1.04 \\
Minimum of ratio $e D / a D$ & 0.50 & 0.69 & 0.76 & 0.92 & 0.67 \\
Maximum of ratio $e D / a D$ & 1.89 & 1.24 & 1.48 & 2.40 & 1.51 \\
Median of $|a D-e D| / a D$ & $19.0 \%$ & $12.8 \%$ & $11.2 \%$ & $32.5 \%$ & $11.3 \%$ \\
Mean of $|a D-e D| / a D$ & $23.0 \%$ & $13.1 \%$ & $14.0 \%$ & $41.9 \%$ & $13.4 \%$ \\
\hline
\end{tabular}

Patients excluded as outliers.

${ }^{\mathrm{a}} e D / a D=16.7$.

${ }^{\mathrm{b}} e D / a D=1.84$.

${ }^{\mathrm{c}}$ One patient was not able to comply with the measurement at $24 \mathrm{~h}$.

deduced from the $24 \mathrm{~h}$ retention and was not observed when the $48 \mathrm{~h}$ retention was used. Only one of the $48 \mathrm{~h}$ retention data published in Hänscheid et al. (2006) yielded an underestimate of $>25 \%$.

In the pooled data from the present investigation and from Hänscheid et al. (2006), the accuracy of estimates based on the $48 \mathrm{~h}$ retention was tested to be: a) not different in groups of hypothyroid patients after thyroid hormone withdrawal and euthyroid patients treated with rhTSH (Wilcoxon rank test: $P=0.73$ ); and b) not dependent on the amount of activity administered (therapeutic versus diagnostic activity, Wilcoxon rank test: $P=0.62$ ).

The dependency of the $48 \mathrm{~h} e D / a D$ values on the blood dose $a D$ is shown in Fig. 2A. An increased probability to overestimate the blood dose is found in patients with $a D<0.09 \mathrm{mGy} / \mathrm{MBq}$ which is not observed at higher specific doses. Relative deviations $(e D-a D) / a D$ are not normally distributed if $a D$ is lower than $0.09 \mathrm{mGy} / \mathrm{MBq}$ (Kolmogorow-Smirnow test: $P<0.01)$. The effect is even more pronounced for dose estimates based on the whole-body residence time according to Equation 3. Although information from additional measurements is enclosed, the accuracy is not superior to that of the single retention formalism because the dose tends to be overestimated if the actual blood dose is small and to be underestimated for higher blood doses (Fig. 2B).

The method presented here is well suited to quantify individual radiation exposure from radioiodine therapy. In most countries, treated patients stay in therapy wards for several days and whole-body activity is routinely monitored. Applying Equation 5, these data can be used to calculate the blood dose as a surrogate for red marrow and organ doses without additional effort.

Deviations between the actual blood dose and the estimate can occur if significantly more or less than
$14 \%$ of $\tau_{\text {total body }}$ is attributed to the blood. Possible situations where this could occur are a relatively large or small initial distribution volume, respectively, or differences in decay characteristics in blood and the whole body due to major activity accumulations in target tissue or the GI-tract. The current study included patients with thyroid remnant uptake values as high as $9 \%$ at $24 \mathrm{~h}$. Unless target tissue uptake is considerably higher than this value, the resulting error potential is mainly due to variations in the initial distribution volume thus bounded by physiological limitations. The ratio of residence times in blood and total body in the patients included in our investigation ranged from 7 to $21 \%$. It seems unlikely that this ratio will be much lower or higher, respectively, in other patients with moderate uptake in target tissue. In the presence of high uptake in accumulating tissue the ratio might be significantly lower if the effective half-life is long and little activity is released back into the blood or significantly higher if much of the activity is released into the blood in form of ${ }^{131}$ I-labeled hormones with long half-life in the circulation. The method has not yet
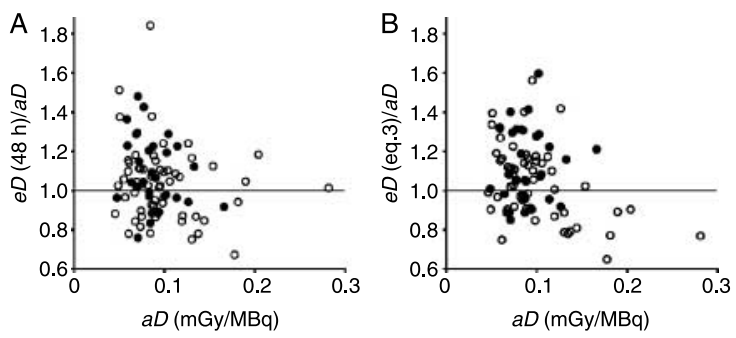

Figure 2 Ratios $e D / a D$ for blood dose estimates $(e D)$ from $A$, a single whole body retention measurement after $t=48 \mathrm{~h}$; and $\mathrm{B}$, from the whole body residence time $\tau_{\text {total body }}$ according to Equation 3 versus actual blood dose $(a D)$. Filled dots represent measurements of patients included in the present investigation $(n=29)$, open circles are data from Hänscheid et al. (2006), $n=59$. 
been validated for patients with large tumors and high uptake and may not be applicable to these patients.

Other uncertainties are of minor importance if major errors introduced by the whole-body activity assessment are ruled out by adequate measuring and quality control procedures (Lassmann et al. 2008).

Choosing the therapeutic activity on the dose to the blood for a given purpose (e.g. ablation of thyroid remnants, treatment of local disease, or distant metastases) is more likely to deliver a consistent activity to the target than current 'one-size fits all' empirically chosen activities do. Notwithstanding that it remains subject to dedicated studies to investigate whether this can improve clinical outcome, a pretherapeutic blood dose estimate from a single wholebody retention can be reliably quantified and used to determine the therapeutic activity to be administered.

The method may not be applicable to high risk patients to be treated with activities aiming at a 2 Gy blood dose. The $2 \mathrm{~Gy}$ limit is likely to be a conservative safety limit, as empiric therapy with $7.4 \mathrm{GBq}$ ( $200 \mathrm{mCi}$ ) has typically been found to be safe despite frequently exceeding the 2 Gy limit to a mild degree, especially in the elderly (Tuttle et al. 2006). However, the present method may underestimate the blood dose in an individual patient by as much as a factor of 0.67 when measured after 1 or 2 days (Table 1). Targeting at 2 Gy might therefore deliver a blood dose of up to $3 \mathrm{~Gy}$ with an increased risk of severe effects on the hematopoietic system, although some have intentionally selected therapeutic activities that deliver this higher dose to the bone marrow (Dorn et al. 2003).

In a conservative approach, to avoid exceeding $2 \mathrm{~Gy}$ to the blood, a maximum target dose of $1.3 \mathrm{~Gy}$ to the blood may be selected based on the minimum observed ratio of estimated to actual blood dose of 0.67 (Table 1). The data shown in Fig. 3 demonstrate that the methodology is applicable to a full range of blood doses and that mostly patients with low specific blood doses due to fast iodine excretion will receive higher activities. The activities determined to deliver $1.3 \mathrm{~Gy}$ to the blood of these patients are often more than three times higher than after application of an empiric standard 7.4 GBq (200 $\mathrm{mCi}$ ) activity with a correspondent increase in tumor dose. Overestimates of the actual doses, which are more common at blood doses $<0.09 \mathrm{mGy} / \mathrm{MBq}$, make this method less than perfect, but do not induce adverse effects on the hematopoietic system as they would result in underdosing the patient, rather than overdosing.

The activities calculated to deliver $1.3 \mathrm{~Gy}$ to the blood were $<7.4 \mathrm{GBq}(200 \mathrm{mCi})$ in only three of 87

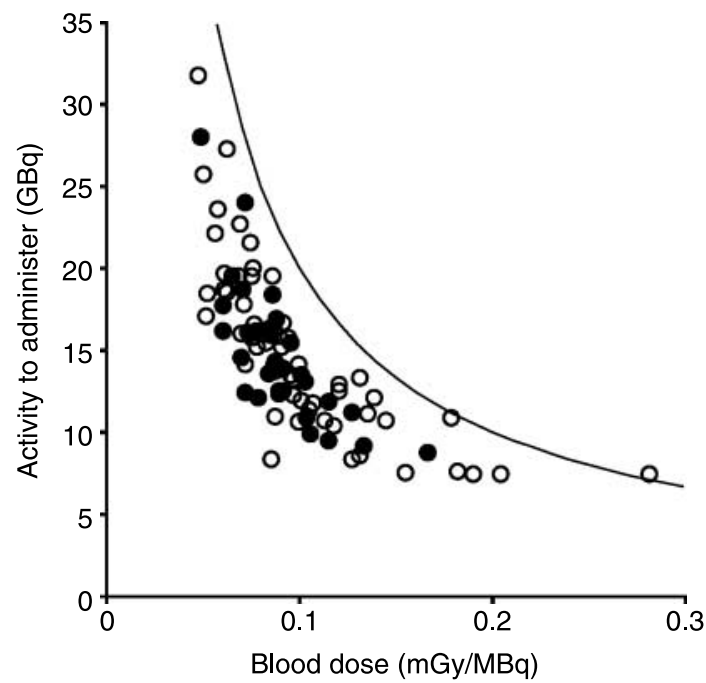

Figure 3 Activities calculated from $48 \mathrm{~h}$ retentions to induce $1.3 \mathrm{~Gy}$ blood dose versus actual specific blood dose. Values were set to the calculated activity or $7.4 \mathrm{GBq}(200 \mathrm{mCi})$, whichever was greatest. Filled dots represent measurements of patients included in the present investigation $(n=29)$, open circles are data from Hänscheid et al. (2006), $n=59$. The curve represents the blood dose limit of $2 \mathrm{~Gy}$.

assessments (three rightmost circles in Fig. 3). An activity of $7.4 \mathrm{GBq}(200 \mathrm{mCi})$ which has been empirically proven to be safe might be administered to these patients for treatment of metastatic disease. The empiric use of $7.4 \mathrm{GBq}$ would have slightly exceeded the limit of 2 Gy to the blood in only one of 87 assessments in this series (rightmost circle in Fig. 3).

A lower targeted blood absorbed dose seems reasonable for remnant ablation therapy in low risk patients. Based on formulas and assumptions presented here, and data derived from a series of patients treated with $3.7 \mathrm{GBq}(100 \mathrm{mCi}$ ) radioiodine (Hänscheid et al. 2006), the mean dose to the blood was 0.45 and $0.29 \mathrm{~Gy}$ when prepared for therapy with hypothyroidism or euthyroidism using rhTSH respectively. As the rate of remnant ablation between the two groups was similar, it may be adequate to routinely select a remnant ablative activity that delivers $0.29 \mathrm{~Gy}$ or, for the sake of simplicity, 0.3 Gy to the blood for patients of similar disease stage. However, as blood dose-based remnant ablation therapy is not yet validated, further investigation will be necessary.

The following example demonstrates how to calculate the blood doses from measured whole-body retention data: two low risk patients present for remnant ablation, and give consent to participate in a study to measure the ablation rate at 0.3 Gy blood dose. The first is a $37-y e a r$ old, $83 \mathrm{~kg}$ (183 pound), $179 \mathrm{~cm}$ tall healthy male with a serum creatinine of $0.81 \mathrm{mg} / \mathrm{dl}$. 
The other is a 79-year old, $97 \mathrm{~kg}$ (214 pound), $178 \mathrm{~cm}$ tall male with multiple medical problems and a serum creatinine of $2.32 \mathrm{mg} / \mathrm{dl}$. What radioiodine activity should be administered? The young man's $46.6 \mathrm{~h}$ whole-body retention was $15.9 \%$, and his blood volume is $5491 \mathrm{ml} \quad(\mathrm{BLV}=31.9 \times$ height +26.3 $\times$ weight -2402 for males). Specific blood dose (Equation 5) is $0.095 \mathrm{mGy} / \mathrm{MBq}$ and $0.3 \mathrm{~Gy}$ to the blood would require $3.2 \mathrm{GBq}(86 \mathrm{mCi})$. Conversely, the older man's $(B L V=5827 \mathrm{ml}) 49.3 \mathrm{~h}$ whole-body retention was $45.8 \%$ yielding $D_{\text {blood }} / A_{0}$ $=0.220 \mathrm{mGy} / \mathrm{MBq}$, and thus to deliver $0.3 \mathrm{~Gy}$ to the blood would require only $1.36 \mathrm{GBq}(37 \mathrm{mCi})$.

\section{Conclusion}

The specific absorbed dose to the blood per unit radioiodine administered to a thyroid carcinoma patient can be determined from a single total-body retention measurement. Accuracy is best when measured after 1 or 2 days, and is sufficient to routinely assess individual radiation exposure from radioiodine therapy and for pre-therapeutic dosimetry in a treatment concept targeting a specific blood dose. Whether or not this approach will yield better clinical outcomes compared to empirically determined radioiodine activities is yet unknown. Given the possibility of underestimating the blood dose by a factor of 1.5 , caution should be used when considering the method to deliver blood doses near the conservative upper limit of 2 Gy blood dose.

\section{Declaration of interest}

The authors declare that there is no conflict of interest that could be perceived as prejudicing the impartiality of the research reported.

\section{Funding}

This research did not receive any specific grant from any funding agency in the public, commercial, or not-for-profit sector.

\section{References}

Benua RS \& Leeper RD 1986 A method and rationale for treating metastatic thyroid carcinoma with the largest safe dose of I-131. In Frontiers in Thyroidology, vol 2, pp 1317-1321. Eds G Meideiros-Neto \& E Gaitan. New York: Plenum.

Benua RS, Cicale NR, Sonenberg M \& Rawson RW 1962 Relation of radioiodine dosimetry to results and complications in treatment of metastatic thyroid cancer. American Journal of Roentgenology 87 171-182.
Dorn R, Kopp J, Vogt H, Heidenreich P, Carroll RG \& Gulec SA 2003 Dosimetry-guided radioactive iodine treatment in patients with metastatic differentiated thyroid cancer: largest safe dose using a risk-adapted approach. Journal of Nuclear Medicine 44 451-456.

Hänscheid H, Lassmann M, Luster M, Thomas SR, Pacini F, Ceccarelli C, Ladenson P, Wahl R, Schlumberger M, Ricard M et al. 2006 Iodine biokinetics and dosimetry in radioiodine therapy of thyroid cancer: procedures and results of a prospective international controlled study of ablation after rhTSH or hormone withdrawal.

Journal of Nuclear Medicine 47 648-654.

Kolbert KS, Pentlow KS, Pearson JR, Sheikh A, Finn RD, Humm JL \& Larson SM 2007 Prediction of absorbed dose to normal organs in thyroid cancer patients treated with ${ }^{131}$ I by use of ${ }^{124}$ I PET and 3-dimensional internal dosimetry software. Journal of Nuclear Medicine 48 143-149.

Lassmann M, Luster M, Hänscheid H \& Reiners C 2004 Impact of ${ }^{131}$ I diagnostic activities on the biokinetics of thyroid remnants. Journal of Nuclear Medicine 4 619-625.

Lassmann M, Hänscheid H, Chiesa C, Hindorf C, Flux G \& Luster M 2008 EANM dosimetry committee series on standard operational procedures for pre-therapeutic dosimetry I: blood and bone marrow dosimetry in differentiated thyroid cancer therapy. European Journal of Nuclear Medicine and Molecular Imaging 35 1405-1412.

Retzlaff JA, Tauxe WN, Kiely JM \& Stroebel CF 1969 Erythrocyte volume, plasma volume, and lean body mass in adult men and women. Blood 33 649-667.

Sgouros G 2005 Blood and bone marrow dosimetry in radioiodine therapy of thyroid cancer. Journal of Nuclear Medicine 46 899-900.

Sgouros G, Kolbert KS, Sheikh A, Pentlow KS, Mun FF, Barth A, Robbins RJ \& Larson SM 2004 Patient specific dosimetry for ${ }^{131}$ I thyroid cancer therapy using ${ }^{124}$ I PET and 3-dimensional-internal dosimetry (3D-ID) software. Journal of Nuclear Medicine 45 1366-1372.

Sisson JC, Shulkin BL \& Lawson S 2003 Increasing efficacy and safety of treatments of patients with well-differentiated thyroid carcinoma by measuring body retentions of ${ }^{131}$ I. Journal of Nuclear Medicine 44 898-903.

Thomas SR, Samaratunga RC, Sperling M \& Maxon HR III 1993 Predictive estimate of blood dose from external counting data preceding radioiodine therapy for thyroid cancer. Nuclear Medicine and Biology 20 157-162.

Traino AC, DiMartino F, Boni G, Mariani G \& Lazzeri M 2004 A minimally invasive method to evaluate ${ }^{131} \mathrm{I}$ kinetics in blood. Radiation Protection Dosimetry 109 249-252.

Tuttle RM, Leboeuf R, Robbins RJ, Qualey R, Pentlow K \& Larson SM 2006 Empiric radioactive iodine dosing regimens frequently exceed maximum tolerated activity levels in elderly patients with thyroid cancer. Journal of Nuclear Medicine 47 1587-1591. 